\title{
On An Extension Of Absolute Cesaro Summability Factor
}

\author{
Aditya Kumar Raghuvanshi, B.K. Singh \& Ripendra Kumar \\ Department of Mathematics IFTM University Moradabad (U.P.) India-244001
}

Abstract: In this paper we have generalised the theorem of Sulaiman which gives some unknown results and known result.s..

Keywords: Absolute summability, increasing sequence, Hölder inequality Minkowski inequality and infinite series.

\section{Introduction}

A positive sequence $\left(b_{n}\right)$ is called an almost increasing sequence if there exist a positive increasing sequence $\left(c_{n}\right)$ and two positive constants $A$ and $B$ such that (Bari [2])

$$
A c_{n} \leq b_{n} \leq B c_{n}
$$

A sequence $\left(\lambda_{n}\right)$ is said to be of bounded variation denoted by $\left(\lambda_{n}\right) \in B V$ if

$$
\sum_{n=1}^{\infty}\left|\Delta \lambda_{n}\right|=\sum_{n=1}^{\infty}\left|\lambda_{n}-\lambda_{n+1}\right|<\infty
$$

A positive sequence $x=\left(x_{n}\right)$ is said to be a quasi- $\sigma$-power increasing seqeuence if there exist a constant $K=K(\sigma, X) \geq 1$ such that $K n^{\sigma} X_{n} \geq m^{\sigma} X_{n}$ holds for all $n \geq m \geq 1$ (Leindler [5]).

Let $\left(\psi_{n}\right)$ be a sequence of complex numbers and let $\Sigma a_{n}$ be a given infinite series with partial sums $\left(s_{n}\right)$ . We denote by $z_{n}^{\alpha}$ and $t_{n}^{\alpha}$ the nth cesaro means of order $\alpha$ with $\alpha>-1$ of the sequence $\left(s_{n}\right)$ and $\left(n a_{n}\right)$ respectively, that is

$$
\begin{aligned}
& z_{n}^{\alpha}=\frac{1}{A_{n}^{\alpha}} \sum_{v=0}^{n} A_{n-v}^{\alpha-1} s_{v} \\
& t_{n}^{\alpha}=\frac{1}{A_{n}^{\alpha}} \sum_{v=0}^{n} A_{n-v}^{\alpha-1} v a_{v}
\end{aligned}
$$

where $A_{n}^{\alpha}=\left(\begin{array}{c}n+\alpha \\ n\end{array}\right)=\frac{(\alpha+1)(\alpha+2) \ldots(\alpha+n)}{n !}=\mathrm{O}\left(n^{\alpha}\right), \mathrm{A}_{-n}^{\alpha}=0$ for $n>0$

The series $\Sigma a_{n}$ is said to be summable $\psi-|C, \alpha|_{k}, k \geq 1$ and $\alpha>-1$ if (Balci [1])

$$
\sum_{n=1}^{\infty}\left|\psi_{n}\left(z_{n}^{\alpha}-z_{n-1}^{\alpha}\right)\right|^{k}=\sum_{n=1}^{\infty} n^{-k}\left|\psi_{n} t_{n}^{\alpha}\right|^{k}<\infty
$$

If $\psi_{n}=n^{1-\frac{1}{k}}$ then $\psi-|C, \alpha|_{k}$-summability is the same as $|C, \alpha|_{k}$-summability (Flett [4]).

\section{Known theorem}

Sulaiman [6] has proved the following theorem.

Theorem 2.1 Let $\left(\psi_{n}\right)$ be a sequence of positive real numbers. Let $\left(X_{n}\right)$ be a quasi- $f$-increasing sequence $f=\left(f_{n}\right), f_{n}=n^{\beta}(\log n)^{\gamma}, 0<\beta \leq 1, \gamma \geq 0$. Let $\left(\lambda_{n}\right)$ and $\left(\mu_{n}\right)$ be sequences of numbers such that $\left(\mu_{n}\right)$ is positive non-decreasing sequences.

If 


$$
\begin{aligned}
& \sum_{n=v}^{m} \frac{\psi_{n}^{k-1}}{n^{k+1}}=\mathrm{O}\left(\frac{\psi_{v}^{k-1}}{v^{k}}\right) \\
& \sum_{n=1}^{\infty} n^{\beta+1}(\log n)^{\gamma} X_{n}\left|\Delta^{2} \lambda_{n}\right|<\infty \\
& \lambda_{n} \rightarrow 0 \text { as } n \rightarrow \infty \quad(2.3) \\
& n^{1+\beta}(\log n)^{\gamma} X_{n} \mu_{n} \Delta\left(\frac{1}{\mu_{n}}\right)=\mathrm{O}(1) \text { as } n \rightarrow \infty \quad(2.4) \\
& \sum_{n=2}^{m} \frac{\psi_{n}^{k-1}\left|t_{n}\right|^{k}}{n^{k}\left(n^{\beta}(\log n)^{\gamma} X_{n}\right)^{k-1}}=\mathrm{O}\left(m^{\beta}(\log m)^{\gamma} X_{m} \mu_{m}\right) \text { an } m \rightarrow \infty \\
& \sum_{n=1}^{m} \frac{\left|\lambda_{n}\right|}{n}<\infty
\end{aligned}
$$

and

$$
\mu_{n} \Delta^{2}\left(\frac{1}{\mu_{n}}\right)=\mathrm{O}\left(\frac{\left|\Delta \lambda_{n}\right|}{n\left|\lambda_{n+1}\right|}\right)
$$

are satisfied then the series $\Sigma a_{n} \lambda_{n} \mu_{n}$ is summable $\psi-(C, 1)_{k}, k \geq 1$.

\section{Main theorem}

In this paper we have proved the following theorem.

Theorem 3.1 Let $\left(\psi_{n}\right)$ be a sequence of complex numbers. Let $\left(X_{n}\right)$ be a quasi- $f$-power increasing sequence, $f=\left(f_{n}\right), f_{n}=n^{\beta}(\log n)^{\gamma}, 0<\beta \leq 1, \gamma \geq 0$. Let $\left(\lambda_{m}\right)$ and $\left(\mu_{n}\right)$ be sequences of the numbers such that $\left(\mu_{n}\right)$ is positive non-decreasing sequence if (2.1), (2.2), (2.3) (2.4), (2.6) (2.7) and

$$
\sum_{n=2}^{m} \frac{\psi_{n}^{k}\left|W_{n}^{\alpha}\right|^{k}}{n^{k}\left(n^{\beta}(\log n)^{\gamma} X_{n}\right)^{k-1}}=\mathrm{O}\left(\left(m^{\beta}(\log m)^{\gamma} X_{m}\right)^{k} \mu_{m}\right)
$$

where $W_{n}^{\alpha}= \begin{cases}\left|t_{n}^{\alpha}\right|, & \alpha=1 \\ \max 1 \leq v \leq n\left|\mathrm{t}_{v}^{\alpha}\right|, & 0<\alpha<1\end{cases}$

Are satisfied then $\sum \frac{a_{n} \lambda_{n}}{\mu_{n}}$ is summable $\psi-|C, \alpha|_{k}, k \geq 1$.

\section{Lemmas}

We have need the following lemmas for the the proof of our theorem.

Lemma 4.1 (Sulaiman [6]) Let $\left(X_{n}\right)$ be a positive non decreasing sequence and, let $\left(\lambda_{n}\right)$ be a sequence of numbers if

$$
\begin{aligned}
& \lambda_{m}=\mathrm{O}(1), \quad m \rightarrow \infty \\
& \left|\lambda_{n}\right| X_{n}=\mathrm{O}(1), \quad n \rightarrow \infty \\
& \sum_{n=1}^{m} n\left|\Delta^{2} \lambda_{n}\right| X_{n}=\mathrm{O}(1) \quad m \rightarrow \infty
\end{aligned}
$$

are satisfied then

$$
\text { neither } \sum_{n=1}^{\infty} \frac{\left|\lambda_{n}\right|}{n}<\infty, \text { nor } \sum_{n=1}^{\infty}\left|\lambda_{n}\right|<\infty
$$

Lemma 4.2 (Sulaiman [6]) Let $\left(X_{n}\right)$ be a quasi- $f$-power increasing sequence 
$f=\left(f_{n}\right), f_{n}=n^{\beta}(\log n)^{\gamma}, 0 \leq \beta \leq 1, \gamma \geq 0$. if

$\lambda_{n} \rightarrow 0$ as $n \rightarrow \infty$

$\sum_{n=1}^{\infty} n^{\beta+1}(\log n)^{\gamma} X_{n}\left|\Delta^{2} \lambda_{n}\right|<\infty$

Then $\quad m^{\beta+1}(\log m)^{\gamma} X_{n}\left|\Delta \lambda_{m}\right|=\mathrm{O}(1)$ as $m \rightarrow \infty$

$\sum_{n=1}^{\infty} n^{\beta}(\log n)^{\gamma} X_{n}\left|\Delta \lambda_{n}\right|=\mathrm{O}(1)$

and $n^{\beta}(\log n)^{\gamma} X_{n}\left|\lambda_{n}\right|=\mathrm{O}(1)$ as $n \rightarrow \infty$.

\section{Proof of the theorem}

Let $\left(T_{n}^{\alpha}\right)$ be the $\mathrm{n}^{\text {th }}(C, \alpha)$ with $0<\alpha \leq 1$, mean of the sequ ence $\left(\frac{n a_{n} \lambda_{n}}{\mu_{n}}\right)$, then

$$
T_{n}^{\alpha}=\frac{1}{A_{n}^{\alpha}} \sum_{v=1}^{n} A_{n-v}^{\alpha-1} \frac{v a_{v} \lambda_{v}}{\mu_{v}}
$$

Applying Abel's transformation and using lemma (4.1), we get that

$$
\begin{aligned}
& T_{n}^{\alpha}=\frac{1}{A_{n}^{\alpha}} \sum_{v=1}^{n-1} \Delta\left(\frac{\lambda_{v}}{\mu_{v}}\right) \sum_{p=1}^{v} A_{n-p}^{\alpha-1} p a_{p}+\frac{\lambda_{n}}{\mu_{n} A_{n}^{\alpha}} \sum_{v=1}^{n} A_{n-v}^{\alpha-1} v a_{v} \\
& \left|T_{n}^{\alpha}\right| \leq \frac{1}{A_{n}^{\alpha}} \sum_{v=1}^{n-1}\left|\Delta\left(\frac{\lambda_{v}}{\mu_{v}}\right)\right|\left|\sum_{p=1}^{v} A_{n-p}^{\alpha-1} p a_{p}\right|+\left|\frac{\lambda_{n}}{\mu_{n}}\right| \frac{1}{A_{n}^{\alpha}}\left|\sum_{v=1}^{n} A_{n-v}^{\alpha-1} v a_{v}\right| \\
& \leq \frac{1}{A_{n}^{\alpha}} \sum_{v=1}^{n-1} A_{v}^{\alpha} W_{v}^{\alpha}\left|\frac{\Delta \lambda_{v}}{\mu_{v}}\right|+\left|\frac{\lambda_{n}}{\mu_{n}}\right| W_{n}^{\alpha} \\
& =\frac{1}{A_{n}^{\alpha}} \sum_{v=1}^{n-1} W_{v}^{\alpha} A_{v}^{\alpha}\left\{\left(\Delta\left(\frac{1}{\mu_{v}}\right) \lambda_{v}+\left(\frac{\Delta \lambda_{v}}{\mu_{v+1}}\right)\right)\right\}+\left|\frac{\lambda_{n}}{\mu_{n}}\right| W_{n}^{\alpha} \\
& =T_{n, 1}^{\alpha}+T_{n, 2}^{\alpha}+T_{n, 3}^{\alpha}
\end{aligned}
$$

To complete the proof of the theorem by minkowskiys inequality, it is sufficient to show that $\sum_{k=1}^{\infty} n^{-k}\left|\psi_{n} T_{n, r}^{\alpha}\right|^{k}<\infty$ for $r=1,2,3$

Now, when $k>1$ applying Hölder's inequality with indices $k$ and $k^{\prime}$, where $\frac{1}{k}+\frac{1}{k^{\prime} 1}=1$ we get that

$$
\begin{aligned}
\sum_{n=2}^{m+1} n^{-k}\left|\psi_{n} T_{n, 1}^{\alpha}\right|^{k} & \leq \sum_{n=2}^{m+1} n^{-k}\left(A_{n}^{\alpha}\right)^{-k}\left|\psi_{n}\right|^{k}\left\{\sum_{v=1}^{n-1} A_{v}^{\alpha} W_{v}^{\alpha}\left|\lambda_{v}\right| \Delta\left|\frac{1}{\mu_{v}}\right|\right\}^{k} \\
& \leq \sum_{n=2}^{m} \frac{\left|\psi_{n}\right|^{k}}{n^{k}}(n)^{-\alpha k} \sum_{v=1}^{n-1}\left|W_{v}^{\alpha}\right|^{k} v^{\alpha k} \Delta\left(\frac{1}{\mu_{v}}\right)\left|\lambda_{v}\right|^{k}\left(\sum_{v=1}^{n-1} \Delta\left|\frac{1}{\mu_{v}}\right|\right)^{k-1} \\
& =\mathrm{O}(1) \sum_{n=2}^{m} \frac{\psi_{n}^{k}}{n^{k(1+\alpha)}} \sum_{v=1}^{n-1} v^{\alpha k}\left|W_{v}^{\alpha}\right|^{k} \Delta\left(\frac{1}{\mu_{v}}\right)\left|\lambda_{v}\right|^{k} \\
& =\mathrm{O}(1) \sum_{v=1}^{m} v^{\alpha k}\left|W_{v}^{\alpha}\right|^{k} \Delta\left(\frac{1}{\mu_{v}}\right)\left|\lambda_{v}\right|^{k} \sum_{n=v}^{m} \frac{\psi_{n}^{k}}{n^{k(1+\alpha)}}
\end{aligned}
$$




$$
\begin{aligned}
& =\mathrm{O}(1) \sum_{v=1}^{m} \frac{v^{\alpha k}\left|W_{v}^{\alpha}\right|^{k}}{v^{k+\alpha k-1}\left(v^{\beta}(\log v)^{\gamma} X_{v}\right)^{k-1}} \Delta\left(\frac{1}{\mu_{v}}\right)\left|\lambda_{v}\right| \psi_{v}^{k}\left(\left.\left|\lambda_{v}\right| v^{\beta}(\log v)^{\gamma} X_{v}\right|^{k-1}\right. \\
& =\mathrm{O}(1) \sum_{v=1}^{m} \frac{v\left|W_{v}^{\alpha}\right|^{k}}{v^{k}\left(v^{\beta}(\log v)^{\gamma} X_{v}\right)^{k-1}} \Delta\left(\frac{1}{\mu_{v}}\right)\left|\lambda_{v}\right| \psi_{v}^{k} \\
& =\mathrm{O}(1) \sum_{v=1}^{m} \frac{\psi_{v}^{k}\left|W_{v}^{\alpha}\right|^{k}}{v^{k}\left(v^{\beta}(\log v)^{\gamma} X_{v}\right)^{k-1}} \Delta\left|\lambda_{v}\right| \Delta\left(\frac{1}{\mu_{v}}\right) \\
& =\mathrm{O}(1) \sum_{n=2}^{m} \frac{\psi_{v}^{k}}{n^{k(1+\alpha)}} \sum_{v=1}^{n-1} v^{\alpha k}\left|W_{v}^{\alpha}\right|^{k} \Delta\left(\frac{1}{\mu_{v}}\right)\left|\lambda_{v}\right|^{k} \\
& =\mathrm{O}(1) \sum_{v=1}^{m}\left(\sum_{r=1}^{v} \frac{\psi_{r}^{k}\left|W_{r}^{\alpha}\right|^{k}}{r^{k}\left(r^{\beta}(\log r)^{\gamma} X_{r}\right)^{k-1}} \Delta\left(v\left|\lambda_{v}\right| \Delta\left(\frac{1}{\mu_{v}}\right)\right)\right. \\
& +\mathrm{O}(1)\left(\sum_{v=1}^{m} \frac{\psi_{v}^{k}\left|W_{v}^{k}\right|^{k}}{v^{k}\left(v^{\beta}(\log v)^{r} X_{v}\right)^{k-1}}\right) m\left(\lambda_{m}\right) \Delta\left(\frac{1}{\mu_{m}}\right) \\
& =\mathrm{O}(1) \sum_{v=1}^{m} v^{\beta}(\log v)^{\gamma} X_{v} \mu_{v}\left(\left|\lambda_{v}\right| \Delta\left(\frac{1}{\mu_{v}}\right)+(v+1)\left|\Delta \lambda_{v}\right| \Delta\left|\frac{1}{\mu_{v}}\right|+(v+1)\left|\lambda_{v+1}\right| \Delta^{2}\left|\frac{1}{\mu_{v}}\right|\right) \\
& +\mathrm{O}(1) m^{\beta+1}(\log m)^{\gamma} X_{m} \mu_{m}\left|\lambda_{m}\right| \Delta\left(\frac{1}{\mu_{m}}\right) \\
& =\mathrm{O}(1) \sum_{v=1}^{m} \frac{\left|\lambda_{v}\right|}{v}+\mathrm{O}(1) \sum_{v=1}^{m-1} v^{\beta}(\log v)^{\gamma} X_{v}\left|\Delta \lambda_{v}\right| \\
& +\mathrm{O}(1) \sum_{v=1}^{m-1} \gamma^{\beta}(\log v)^{\gamma} X_{v}\left|\Delta \lambda_{v}\right|+\mathrm{O}(1) \\
& =\mathrm{O}(1) \\
& \sum_{n=2}^{m} \frac{\left|\psi_{n} T_{n, 2}^{\alpha}\right|^{k}}{n^{k}}=\sum_{n=2}^{m} \frac{\psi_{n}^{k}}{n^{k}}\left|\frac{1}{A_{n}^{\alpha}} \sum_{v=1}^{n-1} A_{v}^{\alpha} W_{v}^{\alpha} \frac{\Delta \lambda_{v}}{\mu_{v+1}}\right|^{k}
\end{aligned}
$$$$
=\mathrm{O}(1) \sum_{n=2}^{m} \frac{\psi_{n}^{k}}{n^{k+\alpha k}} \sum_{v=1}^{n-1} \frac{v^{\alpha k}\left|W_{v}^{\alpha}\right|^{k}}{\left(v^{\beta}(\log v)^{\gamma} X_{v}\right)^{k-1}} \frac{\Delta \lambda_{v}}{\mu_{v+1}^{k}}\left(\sum_{v=1}^{n-1} v^{\beta}(\log v)^{\gamma} X_{v} \Delta \lambda_{v}\right)^{k-1}
$$$$
=\mathrm{O}(1) \sum_{v=1}^{m} \frac{v^{\alpha k}\left|W_{v}^{\alpha}\right|^{k}}{\left(v^{\beta}(\log v)^{\gamma} X_{v}\right)^{k-1}} \frac{\Delta \lambda_{v}}{\mu_{v+1}^{k}} \sum_{n=v}^{m} \frac{\psi_{n}^{k}}{n^{k+\alpha k}}
$$$$
=\mathrm{O}(1) \sum_{v=1}^{m} \frac{v\left|W_{v}^{\alpha}\right|^{k}}{\left(v^{\beta}(\log v)^{\gamma} X_{v}\right)^{k-1}} \frac{\Delta \lambda_{v}}{\mu_{v+1}^{k}} \sum_{n=v}^{m} \frac{\psi_{n}^{k}}{n^{k+1}}
$$$$
=\mathrm{O}(1) \sum_{v=1}^{m} \frac{\left|W_{v}^{\alpha}\right|^{k} \psi_{n}^{k}}{v^{k}\left(v^{\beta}(\log v)^{\gamma} X_{v}\right)^{k-1}} \frac{v\left|\Delta \lambda_{v}\right|}{\mu_{v}}
$$$$
=\mathrm{O}(1) \sum_{v=1}^{m-1}\left(\sum_{r=1}^{v} \frac{\left|W_{r}^{\alpha}\right|^{k} \psi_{r}^{k}}{r^{k}\left(r^{\beta}(\log r)^{\gamma} X_{r}\right)^{k-1}}\right) \Delta\left(\frac{v\left|\Delta \lambda_{v}\right|}{\mu_{v}}\right)
$$$$
+\left(\sum_{v=1}^{m} \frac{\left|W_{v}^{\alpha}\right|^{k} \psi_{v}^{k}}{v^{k}\left(\mathrm{v}^{\beta}(\log )^{\gamma} X_{v}\right)^{k-1}}\right) \frac{m\left|\Delta \lambda_{m}\right|}{\mu_{m}}
$$ 


$$
\begin{aligned}
& =\mathrm{O}(1) \sum_{v=1}^{m-1} v^{\beta}(\log v)^{\gamma} X_{v} \mu_{v}\left(\Delta\left(\frac{1}{\mu_{v}}\right)\left(v\left|\Delta \lambda_{v}\right|+\frac{1}{\mu_{v+1}}\left(\left|\Delta \lambda_{v}\right|+(v+1)\left|\Delta^{2} \lambda_{v}\right|\right)\right)\right) \\
& +\mathrm{O}(1) m X_{m}\left|\Delta \lambda_{m}\right| \\
& =\mathrm{O}(1) \sum_{v=1}^{m-1} v^{\beta}(\log v)^{\gamma} X_{v}\left|\Delta \lambda_{v}\right|+\mathrm{O}(1) \sum_{v=1}^{m-1} v^{\beta}(\log v)^{\gamma} X_{v}\left|\Delta \lambda_{v}\right| \\
& +\mathrm{O}(1) \sum_{v=1}^{m-1} v^{\beta+1}(\log v)^{\gamma} X_{v}\left|\Delta^{2} \lambda_{v}\right|+\mathrm{O}(1) \\
& =\mathrm{O}(1) \\
& \sum_{n=1}^{m} \frac{\left|\psi_{n} T_{n, 3}^{\alpha}\right|^{k}}{n^{k}}=\mathrm{O}(1) \sum_{n=1}^{m} \frac{\psi_{n}^{k}}{n^{k}}\left|\frac{W_{n}^{\alpha} \lambda_{n}}{\mu_{n}}\right|^{k} \\
& =\mathrm{O}(1) \sum_{n=1}^{m} \frac{\psi_{n}^{k}\left|W_{n}^{\alpha}\right|^{k}}{n^{k}\left(n^{\beta}(\log n)^{\gamma} X_{n}\right)^{k-1}} \frac{\left|\lambda_{n}\right|}{\mu_{n}^{k}}\left(n^{\beta}(\log n)^{\gamma} X_{n}\left|\lambda_{n}\right|\right)^{k-1} \\
& =\mathrm{O}(1) \sum_{n=1}^{m} \frac{\psi_{n}^{k}\left|W_{n}^{\alpha}\right|^{k}}{n^{k}\left(n^{\beta}(\log n)^{\gamma} X_{n}\right)^{k-1}} \frac{\left|\lambda_{n}\right|}{\mu_{n}} \\
& =\mathrm{O}(1) \sum_{n=1}^{m-1}\left(\sum_{v=1}^{n} \frac{\left|W_{v}^{\alpha}\right|^{k} \psi_{v}^{k}}{v^{k}\left(v^{\beta}(\log v)^{\gamma} X_{v}\right)^{k-1}}\right) \Delta\left(\frac{v\left|\Delta \lambda_{n}\right|}{\mu_{n}}\right) \\
& +\left(\sum_{n=1}^{m} \frac{\psi_{n}^{k}\left|W_{n}^{\alpha}\right|^{k}}{n^{k}\left(n^{\beta}(\log n)^{\gamma} X_{n}\right)^{k-1}}\right) \frac{\left|\lambda_{m}\right|}{\mu_{m}} \\
& =\mathrm{O}(1) \sum_{n=1}^{m-1} n^{\beta}(\log n)^{\gamma} X_{n} \mu_{n}\left(\Delta\left(\frac{1}{\mu_{n}}\right)\left|\lambda_{n}\right|+\frac{\left|\Delta \lambda_{n}\right|}{\mu_{n+1}}\right)+\mathrm{O}(1) X_{m}\left|\lambda_{m}\right| \\
& =\mathrm{O}(1) \sum_{n=1}^{m-1} \frac{\left|\lambda_{n}\right|}{n}+\mathrm{O}(1) \sum_{n=1}^{m-1} n^{\beta}(\log n)^{\gamma} X_{n}\left|\Delta \lambda_{n}\right|+\mathrm{O}(1) \\
& =\mathrm{O}(1)
\end{aligned}
$$

This completes the proof of theorem.

\section{References}

[1] Balci, M.; Absolute $\psi$-summability factors, Commun. Fac. Sci. Univ. Ank. Ser. A1 Math. Stat. 29, 1980.

[2] Bari, N.K.; Best approximation and differential properties of two conjugate functions, T. Mat. Obs. 5, 1956.

[3] Bor, H.; A new application of quasi power-increasing sequences II, Bor fixed point theory and application, 2013

[4] Flett, T.M.; On an extension of absoulte summability and some theorems of littlewood and paley, Proc. London Math. Soc. 7, 1957.

[5] Leindler, L.; A new application of quasi power increasing sequences, Pub. Math. (Debar.) 58, 2001.

[6] Sulaiman, W.T.; On some generalization of absolute Cesaro summability factors, J. of classical Analysis Vol. 1, 2012. 